\title{
Is Synthetic The New Real? Performance Analysis of Time Series Generation Techniques with Focus on Network Load Forecasting
}

This paper was downloaded from TechRxiv (https://www.techrxiv.org).

\section{LICENSE}

CC BY-NC-SA 4.0

SUBMISSION DATE / POSTED DATE

$20-12-2021 / 22-12-2021$

\section{CITATION}

Naveed, Muhammad Haris; Hashmi, Umair; Tajved, Nayab; Sultan, Neha; Imran, Ali (2021): Is Synthetic The New Real? Performance Analysis of Time Series Generation Techniques with Focus on Network Load Forecasting. TechRxiv. Preprint. https://doi.org/10.36227/techrxiv.17296235.v1

$\mathrm{DOI}$ 


\title{
Is Synthetic the New Real? Performance Analysis of Time Series Generation Techniques with Focus on Network Load Forecasting
}

\author{
Muhammad Haris Naveed, Umair Sajid Hashmi, Member, IEEE, Nayab Tajved, Neha Sultan, \\ and Ali Imran, Senior Member, IEEE
}

\begin{abstract}
The next generation of wireless networks will need to be powered by artificial intelligence (AI) in order to achieve the much needed zero touch automation. However, to achieve this ambitious goal via supervised machine learning, large amounts of training data is required. This training data is publicly unavailable and is a major hindrance in research on AI applications to wireless communication. One way to solve this is to use limited real data to generate synthetic data that can be used in lieu of real data. This can be done using a class of algorithms called generative models. Generative Adversarial Networks (GANs) have been used successfully for this purpose. In this paper, we choose two GAN - based models and one deep learning - based autoregressive model in order to compare their performance at generating synthetic time-series cellular traffic data. We also assess how much real data is required to produce high quality synthetic data. Finally, we compare the performances of traffic forecasting models trained solely on synthetic data, real data, and a mix of both. Our experiments show that the GANs perform better than the autoregressive approach in each aspect considered in this work. DoppelGANger works exceptionally well even in the case of scarce data with difficult seasonality patterns. The TimeGAN struggles to capture long-term seasonality but works very well on short-term trends. Forecasting models trained to predict network load based on data generated by these GANs yield error rates comparable to models trained on real data. Finally, we observe that imputing missing points in the real data with generated data yields minor performance gains in forecasting accuracy.
\end{abstract}

Index Terms-GAN, TimeGAN, PAR, DoppleGANger, Time series, Forecast analysis

\section{INTRODUCTION}

The application of artificial intelligence (AI) in medicine, power systems, image processing and other domains has become commonplace. Although motivated by gains and benefits of zero touch automation presented in earlier studies such as [1], the realization of AI enabled gains in wireless networks is yet to be witnessed in the real world. This is set to change as the world moves to sixth generation networks (6G) and beyond, in which networks will be able to perform self-configuration, self-optimization and self-healing via realtime AI on network parameters [2]. Paired with the rapid proliferation of new and diverse devices, such AR/VR, new connectivity use cases and applications such as IoT, URLLC,

Muhammad Haris Naveed, Umair Sajid Hashmi, Nayab Tajved and Neha Sultan are with the School of Electrical Engineering \& Computer Science, National University of Sciences \& Technology, PK.

Ali Imran is with the AI4Networks Research Center, School of Electrical \& Computer Engineering, University of Oklahoma, OK, USA. and holographic communications, the potential of harnessing the vast data produced by these systems in supervised machine learning (ML) problems to perform usage prediction, optimal resource allocation, anomaly detection and other such applications is substantial [3]-[5]. The ultimate desirable goal is to achieve AI enabled zero touch automation in next generation networks with the aim to minimize operational cost, and overcome operational complexity and human errors thereby maximizing resource efficiency and Quality of Experience.

Unfortunately, sufficient telecommunications network data required for executing sophisticated ML models is either not available, or is too scarce for effective ML model training and execution [6], [7]. This is largely due to privacy concerns and the hesitance of the telecom industry to open source data that could potentially be used by their competition. Another challenge in getting ample training data is the large amount of technical effort required to get data out of silos within the operators where it remains trapped. When data is made available, it is usually locked behind non-disclosure agreements or released to specific research groups. This is a major impediment to research in this domain and partly responsible for the lag in applying ML techniques that we see in the communication systems domain, compared to other domains where data is more freely available, such as image processing.

One solution to this deadlock is to generate synthetic data that is faithful to the properties of the original data, but is different from the original data in terms of actual values. Many techniques to generate data sets of different nature have been suggested over the years. This paper aims to test the latest synthetic data generation techniques based on the generative adverserial networks (GAN) model. GAN is a deep learning based generative model framework that has delivered impressive results in generating synthetic, but near realistic images and videos [8]. We apply multiple variations of the GAN models to two distinct internet activity time-series data sets and compare their performance against a non-GAN based method. We then test the performance of the generated data in downstream supervised Machine Learning applications, specifically future activity prediction. We also analyze how, if at all, is the performance of GANs affected by the amount of data available. While the idea that more data equals better performance is valid, it is interesting to see on how much data can GANs give usable results. Finally, since evaluation of GAN performance is an open problem, and the metrics that 
do exist are geared towards evaluating image output, we use a variety of direct and indirect metrics to comprehensively evaluate the quality of the generated synthetic data.

With this study, we aim to offer the following contributions:

- A performance analysis of GANs designed to generate realistic time-series data on a scarce telecommunications data set is conducted using a select group of indirect metrics, which assess the quality, fidelity and practical usefulness of GAN generated time-series data. We observe that the range of values and structure of a given time-series is retained in the GAN-generated series, but the same cannot be said for non-GAN generated timeseries data.

- Analysis of the impact of data scarcity on the performance of GAN techniques in generating realistic time series synthetic data. While it seems intuitive that increasing the training data would improve the generated data quality, we observe that this is not always the case, and that longer time-series sequences with long-term seasonality are harder for GAN models to learn faithfully.

- Quantitative study of the utility of generated data in downstream predictive modelling using supervised ML approaches. We observe that for simple forecasting purposes, the error between the generated and real data trained model is between 1 to 4 percent for our best performing model.

The paper is organized as follows. Section II discusses relevant literature in this domain, section III describes GAN architectures along with the mathematical description of the individual loss functions. Section IV explains our methodology while section $\mathrm{V}$ explains the experimental setup employed in our simulations. Section VI provides detailed simulation results, and the paper is concluded in Section VII with a summary of the work and future research directions.

\section{RELATED WORK}

The GAN framework was originally designed for simple image data, but since its inception has seen great advancements [8]. CycleGAN [9] deals with image-to-image translation as compared to generating image from a noise vector, with applications such as super resolution and style transfer. StyleGAN [10] focuses on generating high resolution human faces by proposing an alternative generator architecture for GAN based on style transfer learning. StackGAN [11] synthesizes high-quality images from text descriptions using a two-stage process.

Although the extent of applicability of GANs in wireless communications domain is still being explored, however, some recent studies have investigated this particular research theme. To model the channel effects in an end-to-end wireless network in a data-driven way, [12] proposes to use a conditional generative adversarial network (GAN). A novel architecture using GAN in [13] is designed to directly learn the channel transition probability (CTP) from receiver observations. Authors in [14] leverages GAN's ability to generate and discriminate for creating large amounts of synthetic call data records (CDRs), containing the start hour and duration of a call. The authors show a marked improvement in future call duration prediction accuracy using real data augmented with GAN generated syntehtic data points.

There have been GAN based approaches proposed for generating different types of data. Navid Fekri et al. introduces a recurrent generative adversarial network (R-GAN) [15] for generating realistic energy consumption data by learning from real data. Hyland et al. proposed a recurrent conditional GAN (RCGAN) [16] to produce realistic real valued multidimensional medical time series data. Olof Mogren proposes continuous recurrent neural networks (C-RNN) based GAN model [17] that works on sequential data to synthesize music using a long short-term memory (LSTM) NN for both generator and discriminator. Moreover, GANs have also been applied to countless other domains such as finance [18], [19], bio-signals [20], smart grids [21] and data from IoT sensors [22]. A large number of studies have tried to solve the training data scarcity problem using GANs. Changhee Han et al. [23] introduced data augmentation in medical images using GANs. They concluded that data augmentation can boost diagnosis accuracy by $10 \%$. Similar work has been done on images of skin lesions [24] and brain tumor MRIs [25]. Similarly, SimGAN [26] shows a $21 \%$ performance improvement in eyegaze estimation by using an architecture similar to GANs.

Several evaluation techniques have been proposed for data generated by GANs. Ali Borji [27] analyzes more than 24 quantitative and 5 qualitative measures for evaluating generative models. He concludes that each have their own strengths and limitations. He also proposes that the evaluation techniques should be application specific. The most common GAN metrics are Frechet Distance [28] and inception score [29], but many more have been proposed, such as the fidelity and diversity generative model metrics explained in [30]. All in all, as noted in [31], GAN performance evaluation remains an open and challenging research problem.

To the best of our knowledge, our work is the first to compare several modern GAN models vs a non-GAN approach as well as the first to stress test the given models with scarce data. It is also one of the few papers that looks at telecommunications data instead of energy, financial or medical time-series data.

\section{TEleCOM ItAlia DATASET}

\section{A. Background}

As mentioned before, telecommunication data is rarely open-sourced or easily available. When it is available, it is limited to a few research teams that have signed Non Disclosure Agreements with service providers. Telecom Italia (renamed TIM Group in 2019), recognized that this situation hampered independent researchers who couldn't access data for analysis and model training purposes. So in 2014, it organized the 'Telecom Italia Big Data Challenge'. Participants of the Challenge were given accessed to telecommunications, weather, social media, news and electricity consumption data of Milan and the province of Trentino from November 2013 to January 2014, with the non - telecommunication data sets provided by other industrial partners [6]. 
We will be focusing on the telecommunication activity data provided in this set, specifically the call detail records (CDRs), which contain time-series data on internet usage in all regions of the Milan Metropolitan area from November to December 2013. The time range considered in our work is from Monday, November 4, 2013, to Sunday, December 22, 2013. Since the overall challenge made use of data from multiple domains provided by different companies, all of which used different spatial and temporal aggregation methods, Telecom Italia standardised them all onto a single 100x100 grid for Milan, with each square covering about $235 \times 235$ metres of area. The internet usage is measured in 'number of connections created' in 10 minute intervals.

\begin{tabular}{|c|c|c|c|c|}
\hline 9901 & 9902 & $\ldots$ & 9999 & 10000 \\
\hline 9801 & $\ldots$ & $\ldots$ & 9899 & 9900 \\
\hline$\ldots$ & $\ldots$ & $\ldots$ & $\ldots$ & $\ldots$ \\
\hline 101 & 102 & $\ldots$ & $\ldots$ & 200 \\
\hline 1 & 2 & 3 & $\ldots$ & 100 \\
\hline
\end{tabular}

Fig. 1. A sample figure taken from the Telecom Italia dataset [6] which divides the coverage area of Radio Base Station into grids which helps in identifying the geographical location of the user.

\section{B. Regions}

In order to judge how well our selected generation methods work for time-series data of different nature, we look at data from two regions with interesting activity patterns. By 'interesting', we mean that the network activity from these regions contains daily and weekly pattern as well as an overall trend over the selected timespan. These selected regions are

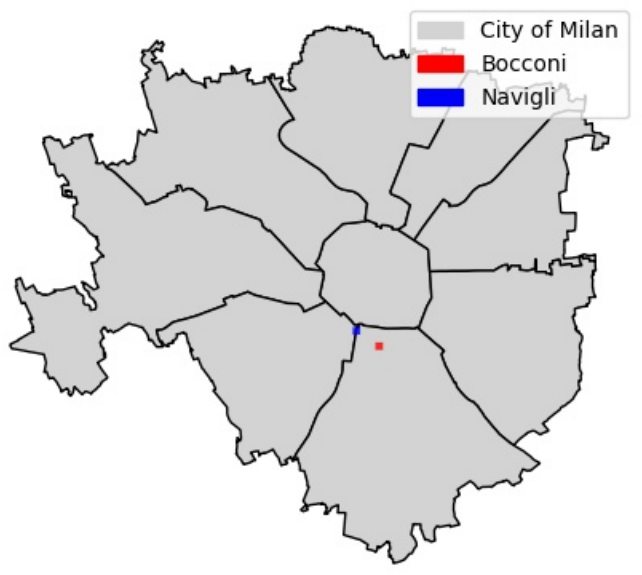

Fig. 2. Map of Milan with our selected areas highlighted.
Bocconi university, a private university, and the Navigli district, a wedge between two canals that boasts upscale restaurants, bars, art galleries and is popular with tourists.

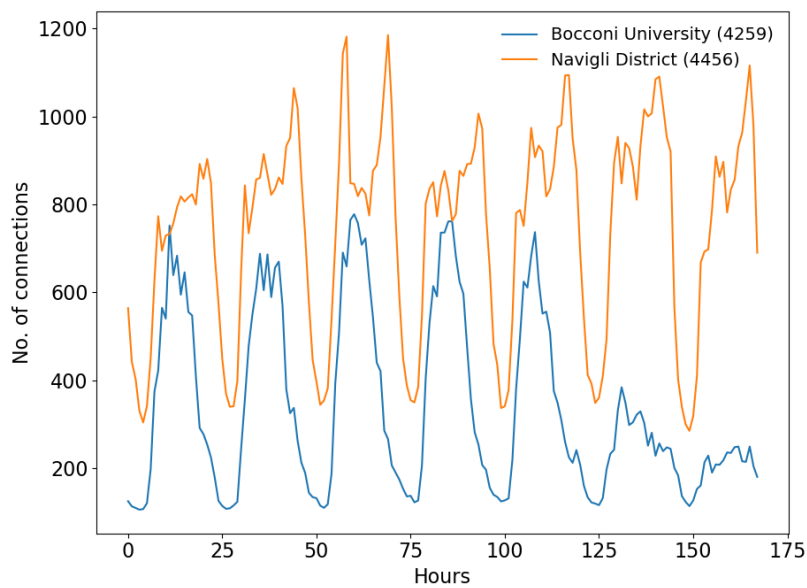

Fig. 3. Internet activity over 1 week (Bocconi and Navigli)

Figs. 1 and 2 illustrate the coverage area and the geographical placement of the selected regions, respectively. Fig. 3 illustrates the activity patterns of each region over a week. We can observe a daily seasonality in Bocconi, with usage peaking around midday and almost disappearing at night. There is an expected decline in internet usage on the weekends, which is expected due to low activity levels in the University campus. In Navigli, we do not observe a dramatic decrease in activity on any particular day, but we observe two peaks per day, one at midday, and a higher one around midnight, indicating the district is a nightlife hotspot. Thus, both regions represent distinct, interesting time-series and it will be interesting to see whether the performance of GANs will differ due to this fact. This is also important since time-series data can take on many different shapes, thus GAN models must be able to perform well across the board in order to be useful.

\section{Time Series Generative Models}

A time series is an ordered sequence of values of a variable taken at equally spaced time intervals. Thus, any analysis or generation of time series must take into account that data points taken over time may have an internal seasonality and trend [32].

Mathematically, most time series' can be written as:

$$
x_{t}=s_{t}+g_{t}+e_{t},
$$

where

- $s_{t}=$ seasonality,

- $g_{t}=$ trend, and

- $e_{t}=$ residuals.

Here, $t=1,2,3, \ldots, N$ represents the time index at which observations have been recorded.

The study and application of time series pervade a large amount of disciplines, including but not limited to finance 
[33], medicine [34], psychology [35] and electrical load management [36]. In this work, we generate network traffic time series data and then test its usefulness in forecasting future network activity patterns. To the best of our knowledge, this is the first attempt to generate network data in this fashion, although building forecasting models for this application has been done extensively before, such as [37] and [38].

We now take a brief look at different methods used to generate time series data, and then focus specifically on the generative models that we have evaluated in our work.

\section{A. Decomposition - based methods}

As shown above, a time-series generally comprises of a seasonality, trend and residual terms. One method of time series generation involves decomposing a time series into its components, then adding a deterministic and stochastic component which are constructed by optimizing weights for the trend and seasonality components and by modelling the residuals via some statistical model [39]. Another approach uses bootstrapping on the residuals obtained after decomposing to create augmented signals and then combines them with trend and seasonality to create a new time series [40].

\section{B. Auto-regressive Models}

Auto-regressive models try to forecast future values of a series based on past values of the same series and a stochastic term. In the simplest auto-regressive generative model, the conditional distributions $p\left(x_{i} \mid x_{<i}\right)$ correspond to a Bernoulli random variable and the model learns a function that maps preceding variables $x_{1}, x_{2}, \ldots x_{i-1}$ to the mean of this distribution [41], resulting in:

$$
p_{q_{i}}\left(x_{i} \mid x_{<i}\right)=\operatorname{Bern}\left(f_{i}\left(x_{1}, x_{2} \ldots x_{i-1}\right)\right),
$$

New data can then be generated by sampling from the conditional distribution learnt by the model. The use of the Bernoulli distribution means that this model cannot learn all types of distributions. This weakness is corrected by models like Neural Auto-regressive Distribution Estimator (NADE) [42], and its extension Real-valued NADE (RNADE) [43], that use neural networks for parameter estimation, which is more efficient than the simple approach described earlier.

Another approach introduced in [44] uses Mixture Autoregressive Models (MAR) to simulate a data set and then fine tunes the fitted MAR models to produce new time series data without any specific features. Deep Auto-Regressive Network describes a generative model that uses an autoencoder whose hidden layers are equipped with autoregressive connections [45]. Popular models based on this principle include WaveNet [46] for audio generation and PixelCNN [47], which generates images pixel by pixel. One major advantage of this class of generative models is that they are simple and their training is stable, although they may not perform as well as other, more complex models like GANs or VAEs.
1) Probabilistic Auto-regressive model (PAR): One implementation of an auto-regressive generative model is PAR, which is a part of the Synthetic Data Vault [48]. PAR uses recurrent neural networks to model the distributions. Since RNNs are designed to handle sequential data, it is much more suited to our task of time series generation.

Given a function $h$ employed within an RNN, we calculate its hidden states $h_{i}=h\left(h_{i-1}, p_{i-1}, \theta\right)$ for each timeinstance, depending on the prior corresponding hidden state $h_{i-1}$, preceding input value $p_{i-1}$ and the network hyper parameters $\theta$. These three variables establishing the hidden state $h_{i}$ construct a set of parameters $\theta\left(h_{i}\right)$ which represent a distribution with density $\theta\left(h_{i}\right)\left(p_{i}\right)$, resulting in the following conditional distribution:

$$
q_{\theta}\left[p_{t_{0}: T} \mid p_{1: t_{0}-1}\right]=\prod_{i=t_{0}}^{T} \ell_{\psi_{h_{i}}}\left(p_{i}\right),
$$

where $q_{\theta}\left[p_{t_{0}: T} \mid p_{1: t_{0}-1}\right]$ is a parametric distribution specified by learn-able parameters $\theta$, which are obtained by using past values $p_{1: t_{0}-1}$ from a time series $p=\left(p_{t}\right) ; 1 \leq t_{0} \leq T$; to forecast future values $p_{t_{0}: T}$ This conditional distribution can then be used to generate new synthetic data similar to the original input data.

We will be evaluating PAR against our chosen GAN based models to evaluate whether the GAN framework yields any noticeable performance improvements against this simpler deep learning-based model. A more comprehensive review of time series data generation techniques, specifically for data augmentation in time series classification and clustering tasks, can be found in [49].

\section{GAN - based methods}

We now move to our main focus, the GAN-based models, in this section, where we will give a brief introduction of the generative adversarial network design, its structure, and its potential issues. First introduced in 2014 [8], generative adversarial networks or GANs consist of two competing agents, typically neural networks, referred to as the generator and discriminator. The generator network attempts to model a noise vector $z$ to fit the probability distribution of the input data, whereas the discriminator attempts to accurately classify the generated data from the real data. Loss convergence of generator and discriminator terminates the training period. Essentially, the two networks are jointly involved in a 2 - player min-max game up until the discriminator fails to distinguish between the real data and the generated data, a point denoted by attainment of Nash equilibrium [50].

Mathematically, this process is expressed as:

$$
\begin{aligned}
\min _{G} \max _{D} V(D, G)= & \mathbb{E}_{x \sim p_{\text {data }(x)}}[\log (D(x)]+ \\
& \mathbb{E}_{z \sim p_{z}(z)}[1-\log (D(G(z))] .
\end{aligned}
$$

Here $x$ is the input data, $\log (D(x))$ is the predicted output of the discriminator for $x_{i}, \log (D(G(z))$ is the output of the discriminator on the GAN generated data $G(z)$. The aim is to maximize the ability of the discriminator to identify real data from generator produced data, so we maximize this value, 
whereas the generator part of the equation tries to minimize the discriminator's ability to correctly classify real and fake data. This translates to maximizing the first term and minimizing the second term of eq. (4). The basic GAN structure is illustrated in Fig. 4.

GANs initially became popular for their ability to produce high-quality image data while avoiding the problems associated it with using Markov chains or approximating unsolvable likelihood functions. This is achieved by training the GANs via backpropagation and using dropout regularization, two methods that have already seen great success in discriminative models. The drawback is that GANs are often hard to train, and suffer from problems like overfitting (reproducing input data), mode collapse (generating samples from only one class in the data) and training instability.

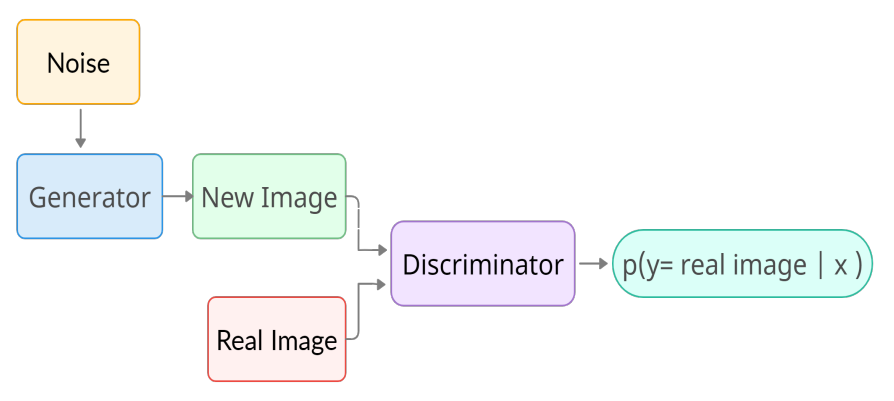

Fig. 4. Simple GAN Structure

The use of GANs to generate tabular or time-series data is less common than generating images or video, with the most popular model (as per GitHub statistics) being the CTGAN (Conditional Tabular GAN) [51]. Another model is the ITSGAN [52], which produces synthetic data tables that exhibit the same statistical properties and functional dependencies as the real, partially available data. MedGAN [53] was the first GAN to model high dimensional, multi-label discrete variables in electronic health records (EHRs). GANs have been used to generate time-series data from many domains such as physiological signals [54], medical ICU data [16], financial time-series [19], [18], and PV (photovoltaic) production [55]. The TimeGAN [56] and DoppelGANger [57] are frameworks that modify the traditional GAN architecture to make it more suitable for time-series data. A brief overview of these models is given below.

1) TimeGAN: The autoregressive models mentioned above are good for capturing the temporal dynamics of a sequence, but are deterministic in nature as opposed to generative. Conversely, GAN architectures such as the RGAN used in [16] do not really take into account the inter-row dependencies of time series data. The TimeGAN solves this problem by combining the GAN framework with an autoregressive setup. Both are trained jointly with the unsupervised GAN loss guided by the supervised autoregressive loss. Additionally, the model makes use of an embedding network to map high level features to a low-level latent feature space. The generator network also first produces samples in the latent space which are then converted back to the original feature space via a recovery network. This is done to reduce high dimensionality in the adversarial learning space. A high level structure of the TimeGAN is shown in Fig. 5.

The recovery and embedding parts are trained via a supervised and reconstruction loss. The reconstruction loss ensures the learnt latent representation is correct and the supervised loss aids the generator in learning the temporal dynamics of the data. The generator and discriminator are then trained in a typical adversarial fashion. Eqs. (5), (6), and (7) detail the reconstruction loss, supervised loss and unsupervised loss of the TimeGAN respectively.

$$
\mathcal{L}_{R}=\mathbb{E}_{s, x_{1}: T \sim p}\left[\|s-\tilde{s}\|_{2}+\sum_{t}\left\|x_{t}-\tilde{x}_{t}\right\|_{2}\right],
$$

where $s$ represent the original static features, $x_{t}$ represent the original temporal features. $\tilde{s}$ and $\tilde{t}$ represent the reconstructions produced by the recovery function.

$$
\mathcal{L}_{S}=\sum_{t}\left[\left\|h_{t}-g_{\chi}\left(h_{s}, h_{t-1}, z_{t}\right)\right\|_{2}\right],
$$

where $h_{t}$ is the true next step latent vector and $g_{\chi}$ represents the approximation of the next step temporal latent vector for a single sample $z_{t}$.

The unsupervised loss is the typical GAN loss function, where we maximize or minimize the likelihood of correct classifications for the discriminator or generator respectively.

$$
\begin{aligned}
\mathcal{L}_{U}= & \mathbb{E}_{s, x_{1}: T \sim p}\left[\log y_{s}+\sum_{t} \log y_{t}\right]+ \\
& \mathbb{E}_{s, x_{1}: T \sim \hat{p}}\left[\log \left(1-\hat{y}_{s}\right)+\sum_{t} \log \left(1-\hat{y}_{t}\right] .\right.
\end{aligned}
$$

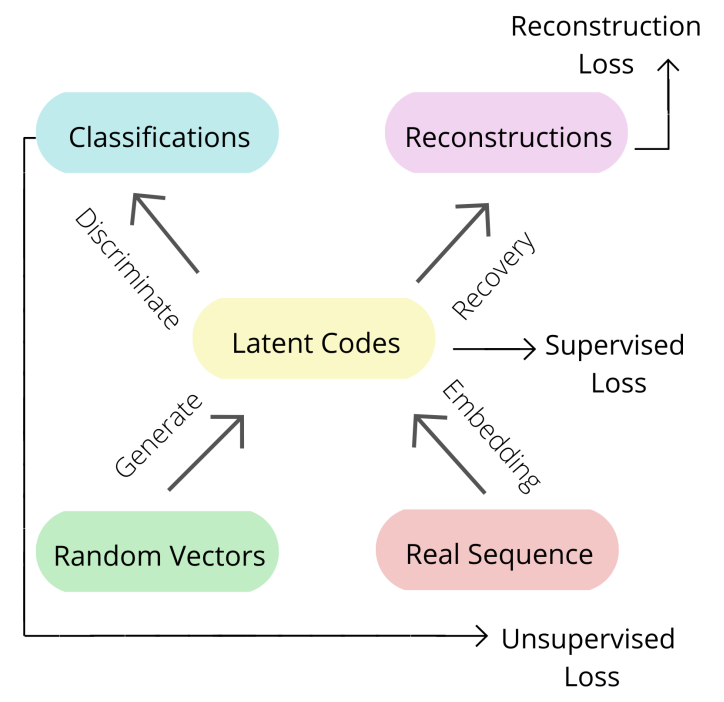

Fig. 5. Block Diagram of component functions in TimeGAN

In the results, unless otherwise stated, the TimeGAN will be referred to as 'tgan.'

2) DoppelGANger: The DoppelGANger introduces several new ideas to solve typical GAN problems like overfitting as well as problems faced when generating longer, more complex time-series data [57]. One change in design, as illustrated in Fig. 6, is how much of the data is generated in a single instance. Since RNNs produce a single measurement in a single pass and for a time-series of length $L$ perform $L$ passes, they tend to forget prior values and struggle to capture long 
term correlations in a time-series. DoppelGANger solves this by modifying the RNN structure to produce $S$ values in a single pass. This reduces the overall number of passes required to generate the entire series, but the quality of the generated samples also deteriorates as $S$ increases. The authors recommend a value of $S=5$ for best results. In order to counter mode collapse, common in data sets that have large variability in values, the authors use an idea they call auto-normalization. Instead of normalizing the entire data set using the minimum and maximum values, they normalize each sample individually and treat the maximum and minimum values of each time series as random data that has to be learnt by the model rather than passed as input. Perhaps the largest design contribution is the use of a separate auxiliary discriminator in addition to the regular generator and discriminator setup, that works only on static features (called metadata) of a single time-series sample. This is done to ensure that the complex relationships between a time-series and its associated metadata are replicated in the generated data. This approach is unique because most models that we have discussed so far trained on the metadata and temporal features jointly. This is also why it uses the Wasserstein Loss as opposed to the regular GAN loss function since the former leads to more stable training in this case. The optimization function for this GAN architecture may be expressed as:

$$
\min _{G} \max _{D_{1}, D_{2}} L_{1}\left(D_{1}, G\right)+L_{2}\left(D_{2}, G\right),
$$

where $L_{i}$ for $\mathrm{i}=1,2$ is the Wasserstein Loss, written as:

$$
\begin{aligned}
L_{i}=\mathbb{E}_{x \sim p_{x}}\left[T_{i}\left(D_{i}(x)\right]-\mathbb{E}_{z \sim p_{z}}\left[D_{i}\left(T_{i}\left(G_{z}\right)\right)\right]-\right. \\
\lambda \mathbb{E}_{\hat{x} \sim \hat{p_{x}}}\left[\left(\| \nabla_{\hat{x}} D_{i}\left(T_{i}(\hat{x}) \|_{2}\right)-1\right)^{2}\right],
\end{aligned}
$$

where $T_{1}(x)=x, T_{2}(x)=t x+(1-t)(G(z))$ and $\mathrm{t}$ is a value from the uniform distribution.

The DoppelGANger will also be referred to as 'DG' in some parts of the paper.A basic block diagram for a DG is illustrated in Fig. 6. A more detailed structure with explanation can be found in Fig.7 of [57].

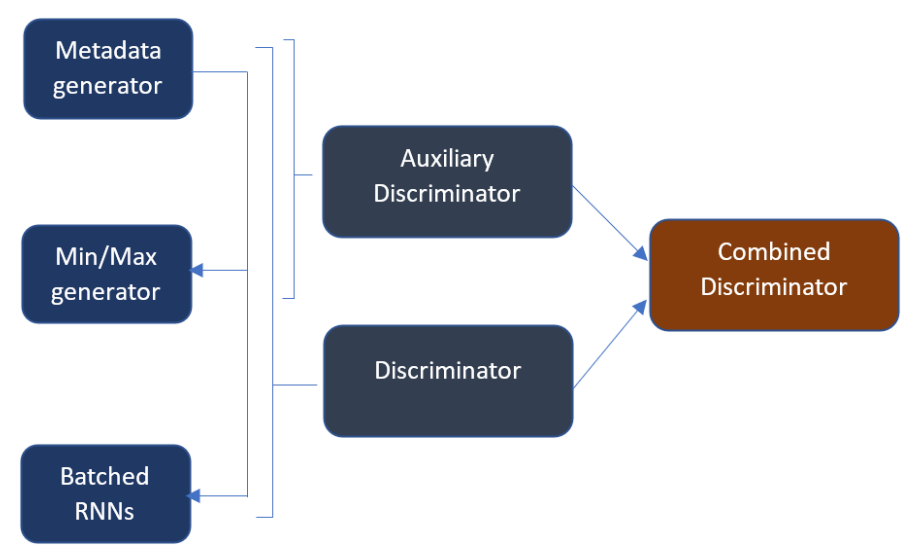

Fig. 6. Simplified block diagram of DoppelGANger

\section{EVAluation Metrics}

Evaluating the quality of GAN generated data is an open research problem. Unlike discriminative models which can be evaluated on fairly robust metrics like accuracy, precision and F1 score among others, generative models have no such counterparts. In the case of images, visual inspection is relied on to determine whether the produced image is good quality. This is not feasible with tabular forms of data. Another method is to indirectly evaluate the quality of the generated data by seeing how well it performs when substituted in place of real data in supervised tasks such as classification and forecasting. Authors in [31] comment on the difficulty of comparing different GAN models and introduce two new metrics that evaluate how well a classifier trained on GAN generated images performs on a test set of real images and vice versa. Other notable metrics used for GANs include Frechet Distance [28] and inception score [29].

Study in [27] provides a comprehensive survey of potential metrics that can be used to evaluate GANs, Most of our chosen metrics indirectly evaluate the quality of the generated data either via use in forecasting models, qualitative assessments, or quantitative measures such as distance. Our criteria for choosing evaluation metrics is based on the following three principles:

- The metrics should favor the generated data that is most similar to the original data.

- The metrics should reward models that generate diverse examples and are not prone to common GAN problems such as overfitting, mode collapse, and mode drop.

- The metrics should be computationally inexpensive and as simple to interpret as possible.

\section{A. Qualitative Assessment}

Perhaps the simplest way to determine how similar our data is to the original, true data is to simply visualize it. While this approach does leave out hard numbers, it gives us a quick high level view of the shape and spread of the data distribution. In this work, we will analyze histograms and auto-correlation function (ACF) plots for this purpose. The histograms will allow us to judge whether the GANs produce data that faithfully captures the range of values present in the original data as well as its distribution. The ACF plots are based on calculating the correlation of a time-series with itself at different, equidistant points in time (referred to as lags). In our case, we choose a lag of up to 24 since we have hourly data and expect network activity patterns to repeat daily. We also ignore all auto-correlation values between +0.05 and 0.05. In general, our aim is to see how similar the ACF plot and histograms of the generated data are to real data.

\section{B. Kullback-Leibler Divergence}

The Kullbeck-Liebler Divergence (or KL - Divergence, or KLD) measures the number of extra bits needed to represent a true distribution $P$ with a code written to represent distribution $Q$ which is an approximation of $P$ [58]. Thus, KL - Divergence can be interpreted as the inefficiency caused by using an approximate distribution $Q$ rather than $P$. Note that this does not mean that KLD is a distance measure; it is not since it is asymmetric and does not obey the triangular inequality. While the use of KL - Divergence is uncommon in comparing 
time-series data, we use it to evaluate the distributions of the two data sets rather than their relationship in time. This can be done by calculating the KLD after discretizing the continuous time-series and using the bin counts to create probability distributions.

The mathematical form of the KLD is shown below:

$$
D_{K L}(P \| Q)=\sum_{x \in X} P(x) \log \frac{P(x)}{Q(x)} .
$$

$\mathrm{P}(\mathrm{x})$ represents our true distribution (the real data), whereas $\mathrm{Q}(\mathrm{x})$ represents the approximate distribution (the generated data). Since the metric is asymmetric, the position of the two series in the formula is important and interchanging the position changes the results.

\section{Dynamic Time Warping (DTW)}

First introduced in 1978, Dynamic Time Warping is a class of algorithms that can be used to compare two ordered sequences against each other [59]. These could be speech sequences, music or any other time ordered sequence. It was originally used in spoken word recognition since it could align the time axis of two sequences, say, the words now and noow and calculate the Euclidean distance between them. This is opposed to directly using a distance metric which would give a large value in the comparison of any two such sequences, even though they are identical. In this work, we employ an R implementation of the algorithm [60].

In mathematical terms, DTW finds a warping function $\phi(k)$ such that,

$$
\phi(k)=\left(\phi_{x}(k), \phi_{y}(k)\right)
$$

where $\phi_{x}$ and $\phi_{y}$ remap the time indexes of the reference series $x$ and test series $y$. Given these warping functions, we find the average distance between these warped $x$ and $y$ series. The aim of the algorithm is to align the two series in such as a way so as to reduce the distance between the series' as much as possible. So the optimization problem can be given as:

$$
D(x, y)=\min _{\phi} d_{\phi}(x, y) \text {. }
$$

The left-over distance is the inherent difference between two sequences. Fig. 7 illustrates how the DTW algorithm aligns two time-series sequences.

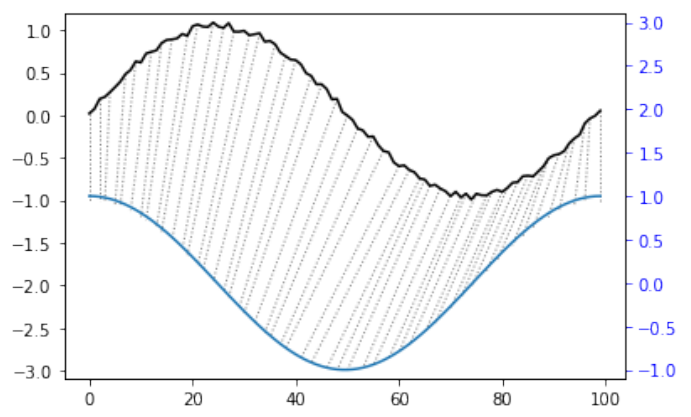

Fig. 7. Aligning two dummy series in DTW [60].

\section{Train Synthetic Test Real (TSTR) and Data Augmenta- tion}

TSTR is an indirect evaluation technique where a predictive model is trained on synthetic data and verified on real data. The dataset generated by GAN is used for training a model which is then tested on examples from real dataset. It was proposed by [16], which assessed a GAN model on a clustering task using a Random Forest Classifier. In our study, however, we use this technique to evaluate the telecommunication data generated by our selected GAN models using a Gradient Boosting Regressor. Firstly, we partition the original dataset into a train and test set. The model is trained on the training set and tested on the held-out test set. This process is Train on Real, Test on Real (TRTR). The test set produced during TRTR is passed to the same model trained on synthetic data. The model's performance is assessed using mean absolute percentage error (MAPE) which has the following mathematical representation:

$$
\operatorname{MAPE}(y, \hat{y})=\frac{100}{n} \sum_{i=1}^{n} \frac{|y-\hat{y}|}{y} .
$$

Here, $y$ and $\hat{y}$ represent the true and forecasted data points, respectively. Additionally, we also augment a small amount of real data with different amounts of synthetic data to study any improvements in forecasting accuracy. The total data is fixed at 5 weeks, but the number of real and synthetic weeks in the data changes. The forecasted week is then compared against the real week to calculate the MAPE.

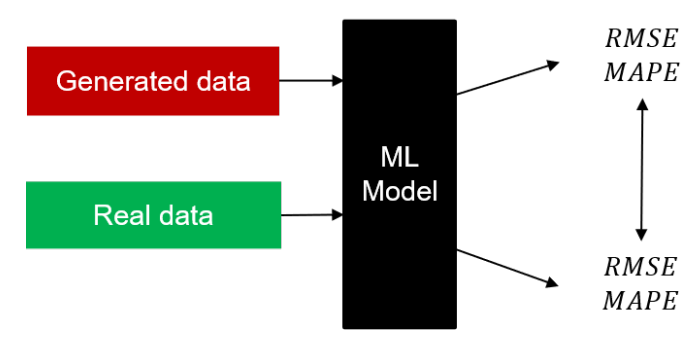

Fig. 8. Block Diagram of the TSTR process.

Following this TSTR pipeline, illustrated in Fig. 8, allows us to compare how much prediction accuracy is affected by replacing the real data with synthetic data in a downstream application such as network load forecasting.

\section{E. Imputation Utility}

In machine learning problems, missing data poses a serious threat to the learning ability of the model. It can introduce bias, make data handling onerous, and reduce efficiency. Missing data is often filled in, or imputed, using a variety of statistical and ML - based techniques. These include: i) carrying the last available reading forward, ii) filling with mean values, iii) imputation via K-nearest neighbours and iv) interpolation [61].

In this work, to introduce scarcity, we delete data from the sequence randomly. After we have obtained our GAN generated data, we impute the missing data using the corresponding sample points from our synthetic data. Similarly, we fill the 
same points via cubic spline interpolation. Finally, we pass the sequence with missing values, the sequence with imputed GAN generated values, and the sequence with interpolated values to the same model and assess how the forecasting accuracy is affected. Fig. 9 shows the imputation utility methodology. The $\% x$ denotes the amount of data removed, which takes on the value of 20,40, 60 and 80 percent.

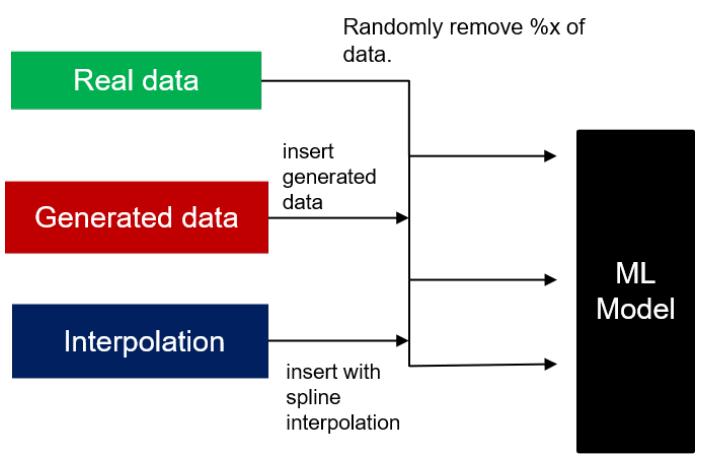

Fig. 9. Block Diagram of the Imputation Utility process

\section{RESUlTS AND DISCUSSION}

\section{A. Pre-processing \& Setup}

We break the original data set into four segments, details of which are given in Table. I. For now, we opted to only work with the univariate internet time-series, though in future we might look at multivariate data as well. For conciseness, we

\begin{tabular}{||c|c|c|}
\hline Number of weeks & timespan & series length \\
\hline \hline 1 & $11 / 4 / 2013-11 / 10 / 2013$ & 168 \\
3 & $11 / 4 / 2013-11 / 24 / 2013$ & 504 \\
5 & $11 / 4 / 2013-12 / 08 / 2013$ & 840 \\
7 & $11 / 4 / 2013-12 / 22 / 2013$ & 1176 \\
\hline
\end{tabular}

TABLE I

DATA PARTITION SCHEME

report the qualitative assessment and DTW of 3, 5 and 7 weeks of data only. For the forecasting analysis, using the complete 7 weeks of data for training would have been inappropriate since the $8^{\text {th }}$ week (which we would be forecasting) would fall into Christmas season, the patterns of which are radically different from our training data. Hence, we train our forecasting model on 1, 3 and 5 weeks of data only and forecasted for a week from $12 / 09 / 2013$ - 12/15/2013. In order to train the model, we extract several features of the time-series by hand, these are briefly explained below:

- T-1: The time-series with lag 1.

- Hours: The hour at which the sample is taken.

- 1st difference: Difference between consecutive timeseries values.

- 2nd difference: Difference between consecutive values of the 1 st difference.

Instead of training the model to predict the value for the next time-step, we train it to predict the difference between the current value and the future time-step. For time-series forecasting, we avoid statistical models like autoregressive integrated moving average (ARIMA) or error trend and seasonality (ETS) since they require significant tuning with domain knowledge of the data set. Also LSTMs or other deep learning based architectures are not employed owing to a lack of training data. Instead, a gradient boosting regressor provided by scikitlearn [62] is used in this work. By using the features above as inputs and training to predict the difference, we are able to 'tabularize' the time-series data and use it to train a decision tree - based algorithm to make forecasts. The model uses 100 estimators, a learning rate of 0.05 and a maximum tree depth of 12 .

\section{B. Qualitative Analysis}

1) Histograms: A quick look at Fig. 10 shows the performance disparity between the three models. It is clear that both DoppelGANger (DG) and TimeGAN (tgan) perform well in terms of capturing the full range of values present in the original data. However, a closer look shows that the TimeGAN struggles to capture outlier values such as the values greater than 700. We see that the DoppelGANger does a slightly better job at capturing less frequent values. The PAR model misses large parts of the original distribution, and even generates negative values at 3 weeks and 7 weeks of data. In general, we see that both TimeGAN and DoppelGANger perform fairly well even with very little training data, and the overlap area between the real and generated distributions increases as we increase the training data from 3 weeks to 7 weeks. This is not the case for PAR, whose performance remains much the same even as we increase the available data.

These observations are more marked in a similar analysis of the Navigli area, shown in Fig. 11. We observe a larger overlap between the two distributions as the training data is increased, except for PAR, whose performance seems unaffected. In this case, however, the DoppelGANger does miss more outlying values in the original data, although it still captures them better than the TimeGAN.

2) ACF Plots: While the histograms give an idea about the distribution of the values of a time-series, they do not provide any information on how well the generated series captures the temporal correlation of the original series. For this, we create a series of auto-correlation function (ACF) plots in a similar fashion to the histograms. Fig. 12 shows the ACF plots for Bocconi region. The plots depict a slightly better performance of the DoppelGANger than the TimeGAN in capturing the extreme correlation peaks. However, PAR fails to capture the temporal dependence between the days of a week at all.

The same idea is repeated in the Navigli district in Fig. 13, where the performance of the TimeGAN deteriorates compared to that of DoppelGANger, while PAR again struggles with capturing temporal dynamics of the input time-series. In general, we see that the DoppelGANger captures temporal dependencies much better than either of the other two models, while PAR produced time-series may lie in the same range of values, but fails to adequately capture the underlying temporal structure of the series.

Surprisingly, the performance of the models seems to deteriorate as we increase the available training data. This 
Bocconi University
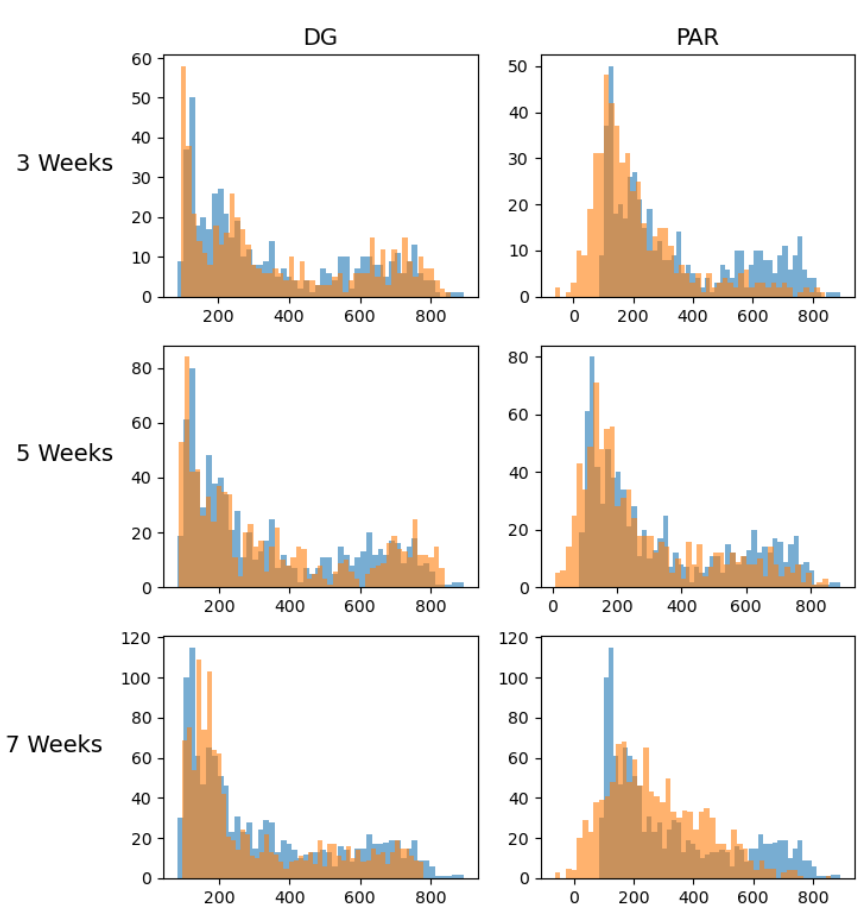
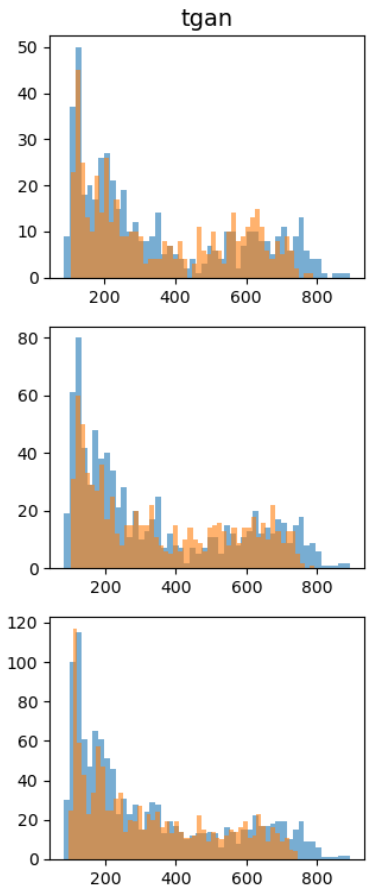

Fig. 10. Distributions of GAN generated data based on amount of data on which the GAN was trained (Bocconi University).

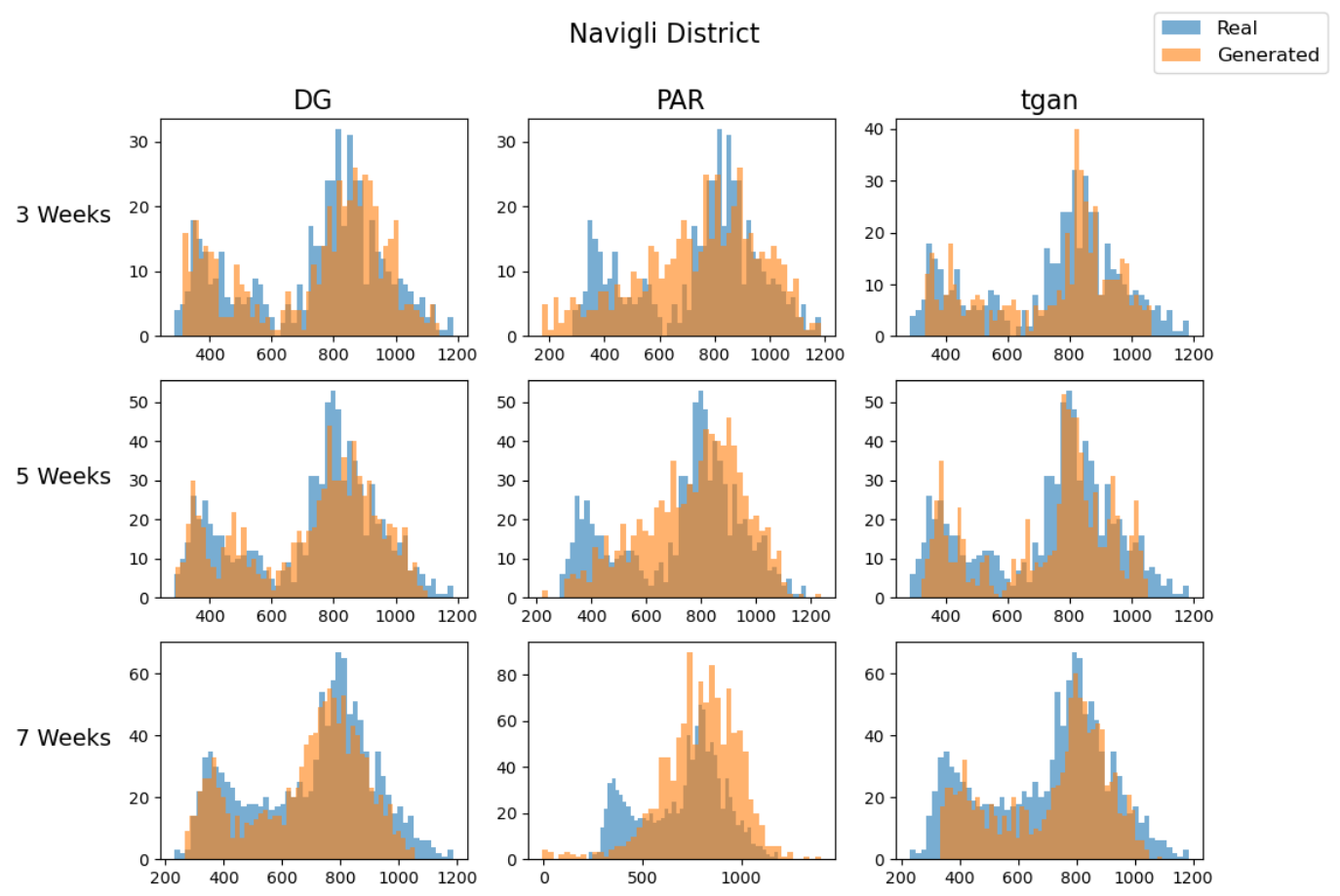

Fig. 11. Distributions of GAN generated data based on amount of data on which the GAN was trained (Navigli district). 


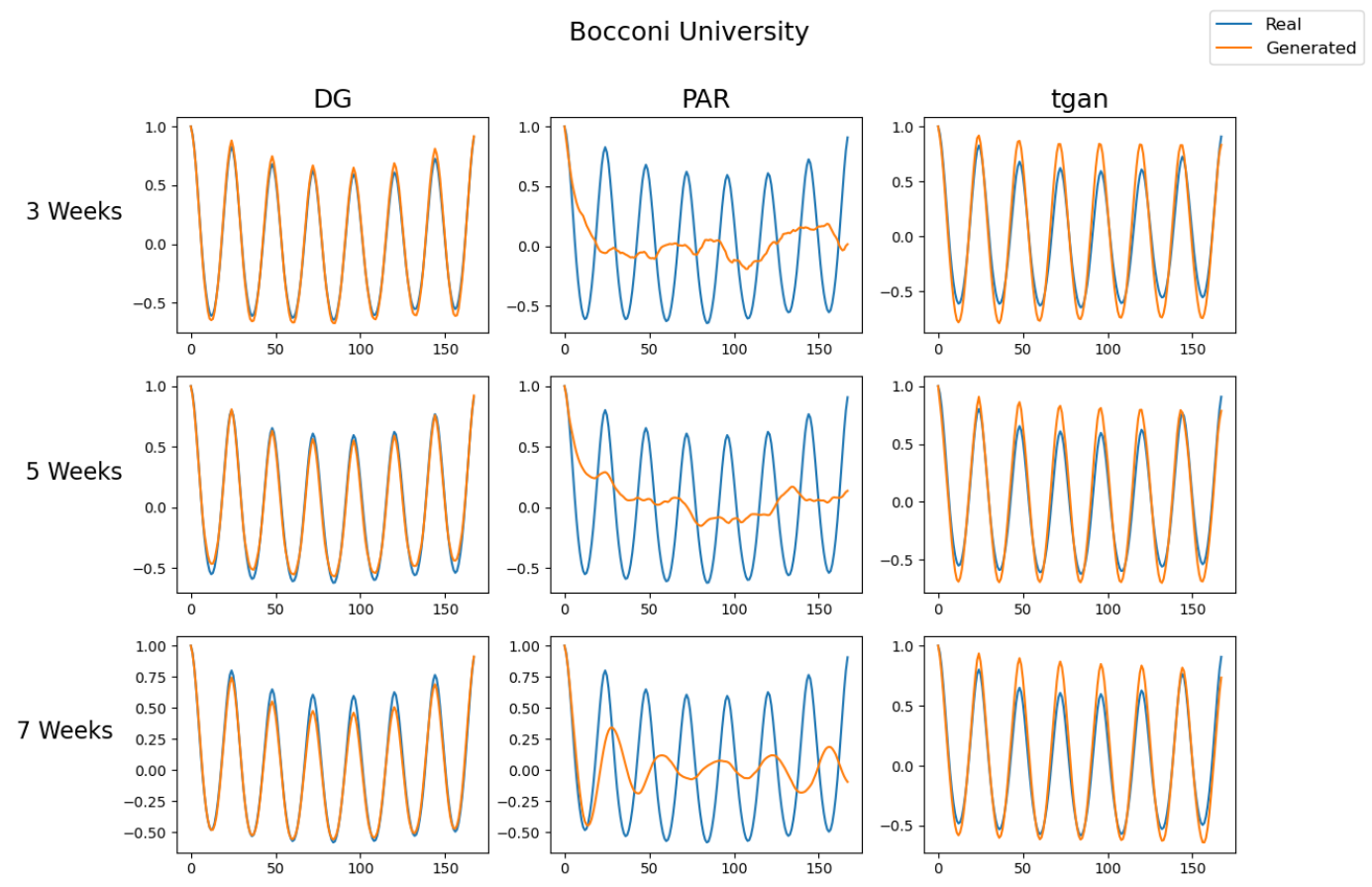

Fig. 12. ACF plots of real vs generated data (Bocconi university).

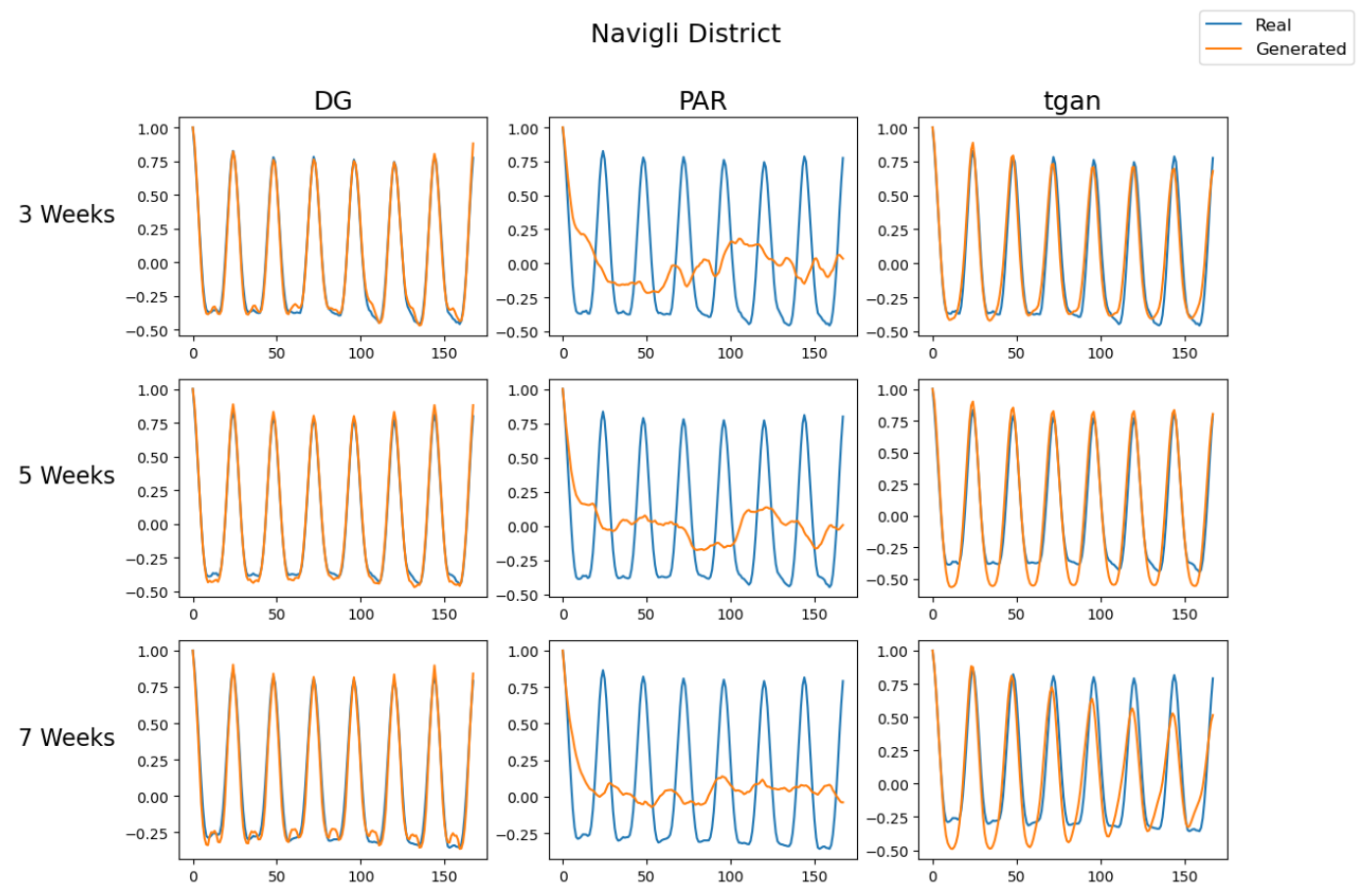

Fig. 13. ACF plots of real vs generated data (Navigli District). 
can be for two reasons: firstly, the amount of data added is simply not enough to have a significant improvement in performance; time-series data usually spans years instead of a few weeks. Secondly, increasing the data increases the number of long-term dependencies that each model has to learn. As previously explained, holding these dependencies in memory is difficult for RNN - based architectures, but the DoppelGANger's superior performance indicates that it's solution for this problem does improve performance over standard RNNs (as used in PAR) or RNNs used in conjunction with auto-regressive models (as used in TimeGAN).

\section{Quantitative Analysis}

Now that we have a cursory idea of the quality of the generated data, we will quantify its usefulness through some direct and indirect metrics which have already been explained in the preceding sections.

1) Dynamic Time Warping: The DTW results in Tables II and III reveal that the DoppelGANger's performance is marginally better than TimeGAN, although this margin seems to increase in favor of the DoppelGANger as the amount of available data increases. Note that the comparison is between 1 week of generated data vs 1 week of the input real data and so on. We notice only minor fluctuation in the values from Bocconi, with the exception of PAR, whose score improves as we increase data. This is because there are more random points in 7 weeks of PAR data that happen to align well with the real series than at 3 or 5 weeks. This unique trend is not seen for TimeGAN or DoppelGANger, although both are better performing than PAR. Their scores remain roughly constant as the available data increases.

The large disparity between the performance of DoppelGANger and TimeGAN for Bocconi University (where the DoppelGANger is easily superior) and the Navigli district, where the performance of both GANs is fairly even can be explained by plotting the generated time-series of both vs the original series, as done in Fig. 14. The figure shows how the TimeGAN manages to capture the cyclical pattern but does not capture the sharp downturns in internet usage on the weekends. This pattern is learned by the DoppelGANger, resulting in a lower alignment cost with the original series.

\begin{tabular}{||c|c|c|c||}
\hline \multicolumn{4}{|c|}{ Bocconi University } \\
\hline Number of weeks & DoppelGANger & PAR & TimeGAN \\
\hline 3 & 22.2 & 55.79 & 30.65 \\
7 & 23.48 & 50.9 & 36.7 \\
& 21.7 & 42.25 & 31.47 \\
\hline \multicolumn{3}{|c}{ TABLE II }
\end{tabular}

DTW - BASED SIMILARITY SCORES (LOWER THE BETTER) FOR BOCCONI UNIVERSITY

2) KL - Divergence: The KL - Divergence calculations for Bocconi and Navigli are shown in Tables IV and V, respectively. There is no significant pattern to be seen, other than that differences for DoppelGANger and TimeGAN tend to stay consistent, whereas PAR generated data outputs the largest values and demonstrates the most volatility. In general, from an information theory perspective, we would not need a

\begin{tabular}{||c|c|c|c||}
\hline \multicolumn{4}{|c|}{ Navigli District } \\
\hline Number of weeks & DoppelGANger & PAR & TimeGAN \\
\hline 3 & 28.75 & 64.93 & 32.37 \\
7 & 28.88 & 56.66 & 34 \\
7 & 31.25 & 61.65 & 34.26 \\
\hline \multicolumn{4}{|c}{ TABLE III }
\end{tabular}

DTW - BASED SIMILARITY SCORES (LOWER THE BETTER) FOR NAVIGLI DISTRICT
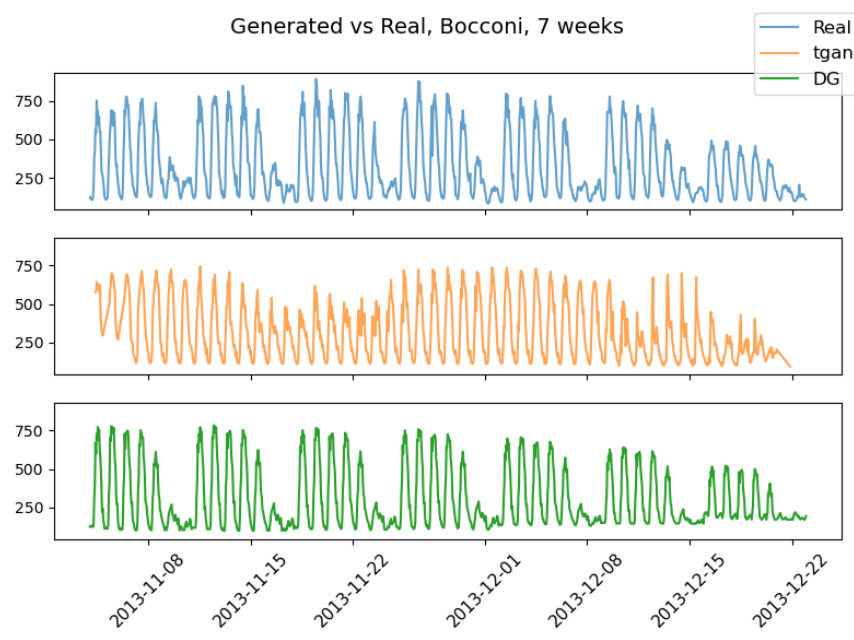

Fig. 14. Generated vs Real, Bocconi, 7 Weeks for DG and tgan.

great many more bits to represent the generated distribution as the real distribution.

3) Train Synthetic Test Real (TSTR): Tables VI and VII list the TSTR results (forecasting accuracy in MAPE) for Bocconi and Navigli on the test period spanning one week from December 9 to December 15.

The fairly low MAPE at data as little as 1 week immediately stands out. Unfortunately, this is a result of scarce data, because of which the test data that we have heavily resembles the original training data, so better performance is unsurprising. This performance improves as one would expect with increasing the available training data, but this behavior is not observed in the generated data of any model. Infact, their performance deteriorates as more data is made available, indicating that the later weeks add distortion that does not help the model. In any case, the DoppelGANger's performance is still the best amongst all models. Surprisingly, PAR performs better than the TimeGAN. This is probably because PAR captures, even if unfaithfully, the drops in activity on weekends, whereas the TimeGAN merely recreates a sinusoidal wave.

We observe some of the trends highlighted in the last paragraph for Navigli as well. The largest difference is that PAR's performance is now clearly worse than the other two models. Fig. 15 shows a comparison between the true and predicted test data of a model trained on synthetic TimeGAN data.

We can conclude based on the low error differences between the DoppelGANger and TimeGAN trained models, as well as the model trained on original data that generated data can be a good substitute for true data in situations where the real data 


\begin{tabular}{||c|c|c|c||}
\hline \multicolumn{4}{|c|}{ Bocconi University } \\
\hline Number of weeks & DoppelGANger & TimeGAN & PAR \\
\hline 3 & 0.04 & 0.02 & 0.1 \\
5 & 0.01 & 0.03 & 0.014 \\
7 & 0.036 & 0.034 & 0.1 \\
\hline \multicolumn{4}{|c}{ TABLE IV } \\
\hline
\end{tabular}

KL - DIVERGENCE (LOWER THE BETTER) FOR BOCCONI UNIVERSITY

\begin{tabular}{||c|c|c|c||}
\hline \multicolumn{4}{|c|}{ Navigli District } \\
\hline Number of weeks & DoppelGANger & TimeGAN & PAR \\
\hline 3 & 0.01 & 0.009 & 0.02 \\
5 & 0.016 & 0.004 & 0.06 \\
7 & 0.013 & 0.017 & 0.233 \\
\hline
\end{tabular}

KL - DiVERgENCE (LOWER THE BETTER) FOR NAVIGLi DisTRICT

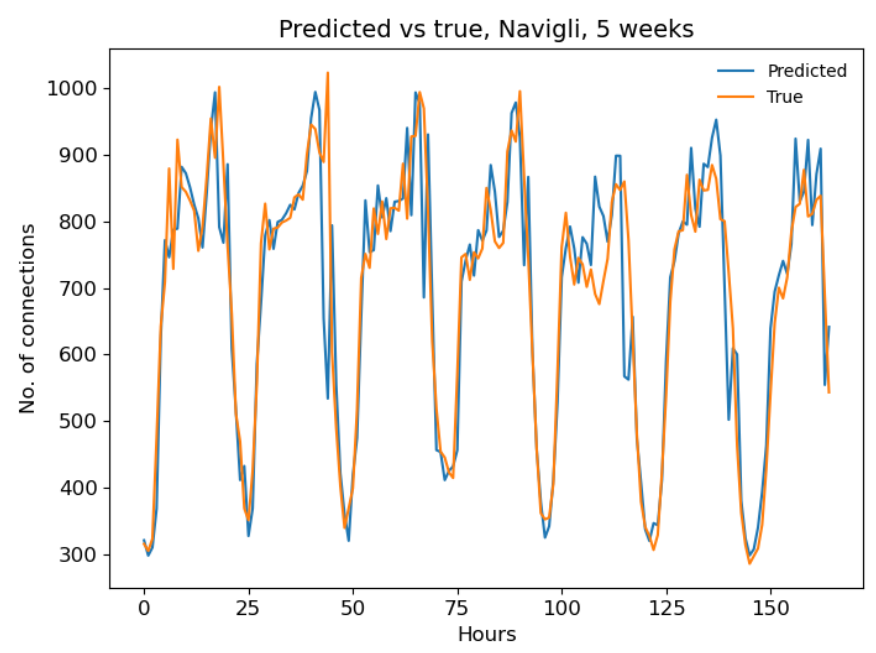

Fig. 15. True vs predicted, Navigli, trained on 5 weeks of TimeGAN data.

cannot be shared easily due to privacy policies or other legal complications.

\begin{tabular}{||c|c|c|c|c||}
\hline \multicolumn{5}{|c|}{ Bocconi University } \\
\hline Train data (in weeks) & DoppelGANger & TimeGAN & PAR & TRTR \\
\hline 1 & 10.47 & 15.72 & 13.46 & 10.48 \\
3 & 12.54 & 16.54 & 16.24 & 8.47 \\
5 & 12.31 & 16.21 & 14.83 & 7.26 \\
\hline
\end{tabular}

TABLE VI

Train Synthetic Test Real (TSTR) AND Train Real TeSt Real (TRTR) PREDICTION ACCURACIES (IN MAPE) FOR BOCCONI UNIVERSITY

\begin{tabular}{||c|c|c|c|c||}
\hline \multicolumn{5}{|c|}{ Navigli District } \\
\hline Train data (in weeks) & DoppelGANger & TimeGAN & PAR & TRTR \\
3 & 7.44 & 10.33 & 14.92 & 7.34 \\
5 & 6.85 & 7.89 & 10.13 & 7.20 \\
5.85 & 8.1 & 12.08 & 6.84 \\
\hline
\end{tabular}

TABLE VII

Train Synthetic Test Real (TSTR) and Train Real Test Real (TRTR) PREDICTION ACCURACIES (IN MAPE) FOR NAVIGLI DISTRICT

4) Performance with Augmented Data Set: Augmenting the real data with synthetic data yields results shown in Tables VIII and IX. We see this yields no improvement in the case of

\begin{tabular}{||c|c|c|c||}
\hline \multicolumn{4}{|c|}{ Bocconi University } \\
\hline real + synthetic (weeks) & DoppelGANger & TimeGAN & PAR \\
\hline $1+4$ & 9.63 & 15.04 & 11.57 \\
$4+1$ & 7.46 & 8.46 & 8.23 \\
$2+3$ & 8.95 & 11.27 & 10.4 \\
$3+2$ & 7.28 & 7.70 & 8.03 \\
\hline
\end{tabular}

TABLE VIII

AUGMENTED DATA PERFORMANCE FOR BOCCONI UNIVERSITY (TRTR FOR 5 WEEKS' REAL DATA IS $7.26 \%$ ). ALL VALUES ARE IN MAPE

\begin{tabular}{||c|c|c|c|}
\hline \multicolumn{3}{|c|}{ Bocconi University } \\
\hline real + synthetic (weeks) & DoppelGANger & TimeGAN & PAR \\
\hline $1+4$ & 7.22 & 7.77 & 9.1 \\
$4+1$ & 6.61 & 7.07 & 6.98 \\
$2+3$ & 7.17 & 7.43 & 8.31 \\
$3+2$ & 7.1 & 6.53 & 7.01 \\
\hline
\end{tabular}

TABLE IX

AUGMENTED DATA PERFORMANCE FOR NAVIGLI DISTRICT (TRTR FOR 5 WEEKS' REAL DATA IS 6.84\%). ALL VALUES ARE IN MAPE.

Bocconi, but minor improvements, such as when 4 weeks of real data is combined with 1 week of DoppelGANger generated data to improve the error by $0.23 \%$. A similar improvement of $0.3 \%$ is seen when combining 3 weeks of real data with 2 weeks of TimeGAN generated data. Neither of these performance gains is significant but indicate that larger amounts of synthetic data could yield more reliable performance gains.

5) Imputation Utility: Tables $\mathrm{X}$ and $\mathrm{XI}$ display the prediction errors for five models trained on mixed data from all three generative models, as well as interpolated data. For comparison, we also train a model on the smaller data set created by randomly removing observations. We see that the performance of all models deteriorate as we use them to fill larger gaps in the real data set. However, the DoppelGANger's performance appears the most stable, followed by the TimeGAN although it exhibits higher errors overall. We also observe the model trained on PAR imputed data performs worse than a model trained on true unimputed data, but its performance suddenly improves when the original data is reduced by $90 \%$, similar to what we observed in Table. VI. We can now conclude that PAR's uncharacteristically good performance in this case is probably due to our model learning some spurious correlations that happen to allow it to predict well on the test data despite the poor training data. The Navigli district recordings show similar trends, except that the TimeGAN and DoppelGANger perform equally well, and the gap between the GAN imputed data sets and the interpolated data is wider, at around $4 \%$.

While we notice the DoppelGANger imputed model performs well, simply interpolating over the missing data with a common technique like cubic splines seems to impute the data just as well. The interpolated model's performance deteriorates at $80 \%$ and $90 \%$ missing data levels, where the DoppelGANger imputed data yields a roughly $2.5 \%$ accuracy gain. The results emphasize that GAN based models really start yielding true benefits at high sparsity levels, where interpolation schemes fade off. Other works such as [63] and [64] have also successfully demonstrated the use of GANs for time series imputation.

The key takeaway here is that the DoppelGANger's perfor- 


\begin{tabular}{||c|c|c|c|c|c||}
\hline \multicolumn{6}{|c|}{ Bocconi University } \\
\hline \%age data removed & DoppelGANger & TimeGAN & PAR & Missing & Interpolated \\
\hline 20 & 7.22 & 11.25 & 14.61 & 10.62 & 7.72 \\
60 & 8.23 & 14.25 & 19.84 & 16.83 & 8.36 \\
80 & 9.99 & 15.45 & 24.24 & 31.67 & 10.34 \\
90 & 11.51 & 14.28 & 21.71 & 53.91 & 13.23 \\
& 11.35 & 14.98 & 15.13 & 54.04 & 13.93 \\
\hline
\end{tabular}

TABLE X

IMPUTING MISSING DATA VIA INTERPOLATION AND WITH GENERATED DATA FOR BOCCONI UNIVERSITY. ALL VALUES ARE MAPE

\begin{tabular}{||c|c|c|c|c|c||}
\hline \multicolumn{7}{|c||}{ Navigli District } \\
\hline \%age data removed & DoppelGANger & TimeGAN & PAR & Missing & Interpolated \\
\hline 20 & 6.88 & 6.54 & 9.91 & 8.42 & 7.08 \\
40 & 6.36 & 7.47 & 18.71 & 9.46 & 6.9 \\
60 & 7.02 & 7.94 & 21.66 & 16.08 & 7.76 \\
80 & 6.94 & 8.74 & 22.83 & 27.65 & 11.57 \\
90 & 7.24 & 7.8 & 14.85 & 27.74 & 11.31 \\
\hline
\end{tabular}

TABLE XI

IMPUTING MISSING DATA VIA INTERPOLATION AND WITH GENERATED DATA FOR BOCCONI UNIVERSITY FOR NAVIGLI DISTRICT. ALL VALUES ARE MAPE

mance for different types of time series data is more reliable, since using it to impute random gaps in the true data yields the most accuracy, in some cases outperforming the model trained entirely on true data. The TimeGAN performs well for Navigli owing to its repetitive pattern with no sharp changes, but does relatively poorly in Bocconi. Finally, PAR's output is more or less random, and it only captures the value distribution of the original series reasonably well.

\section{CONCLUSION}

In this work, we compared three time-series generative models against each other using an actual mobile network data set. Two methods are based on the GAN - architecture, while one is a deep learning based auto-regressive model. We see that the GAN based architectures are superior to the autoregressive approach across an array of numerical and graphical measures. We used the generated data to train a supervised machine learning algorithm and assessed its performance on unseen real data. These experiments revealed that the GAN based DoppelGANger and TimeGAN generated data yielded models were competitive with a model trained on true data. Finally, our simulations revealed that increasing the training data incrementally did not yield any benefits but instead degraded the generative model's performance. This implies that to get any substantial performance improvements by increasing training data, we must increase it substantially. Nevertheless, the chosen GAN models, in particular the DoppelGANger performed very well even on sparse data set. While this is the first indepth research on GAN model comparison for a tabular telecommunications data set, future extensions of the work may involve replicating with a much larger data set, as well as with multivariate time-series data.

\section{ACKNOWLEDGMENT}

This work was supported in part by the National Science Foundation (NSF) under Grant 1619346, and Grant 1923669; and in part by the Qatar National Research Fund (QNRF) under Grant NPRP12-S 0311-190302.

\section{REFERENCES}

[1] A. Imran, A. Zoha, and A. Abu-Dayya, "Challenges in 5G: how to empower SON with big data for enabling 5G," IEEE Network, vol. 28, no. 6, pp. 27-33, 2014

[2] J. Moysen and L. Giupponi, "From 4G to 5G: Selforganized network management meets machine learning," Computer Communications, vol. 129, pp. 248-268, 2018. [Online]. Available: https://www.sciencedirect.com/science/article/pii/S0140366418300380

[3] U. S. Hashmi, A. Darbandi, and A. Imran, "Enabling proactive selfhealing by data mining network failure logs," in 2017 International Conference on Computing, Networking and Communications (ICNC), 2017, pp. 511-517.

[4] U. S. Hashmi, A. Rudrapatna, Z. Zhao, M. Rozwadowski, J. Kang, R. Wuppalapati, and A. Imran, "Towards Real-Time User QoE Assessment via Machine Learning on LTE Network Data," in 2019 IEEE 90th Vehicular Technology Conference (VTC2019-Fall), 2019, pp. 1-7.

[5] M. Ibrahim, U. S. Hashmi, M. Nabeel, A. Imran, and S. Ekin, "Embracing Complexity: Agent-Based Modeling for HetNets Design and Optimization via Concurrent Reinforcement Learning Algorithms," IEEE Transactions on Network and Service Management, vol. 18, no. 4, pp. 4042-4062, 2021.

[6] G. Barlacchi, M. De Nadai, R. Larcher, A. Casella, C. Chitic, G. Torrisi, F. Antonelli, A. Vespignani, A. Pentland, and B. Lepri, "A multi-source dataset of urban life in the city of Milan and the Province of Trentino," Scientific Data, vol. 2, 2015.

[7] D. Gündüz, P. de Kerret, N. D. Sidiropoulos, D. Gesbert, C. R. Murthy, and M. van der Schaar, "Machine Learning in the Air," IEEE Journal on Selected Areas in Communications, vol. 37, no. 10, pp. 2184-2199, 2019.

[8] I. Goodfellow, J. Pouget-Abadie, M. Mirza, B. Xu, D. Warde-Farley, S. Ozair, A. Courville, and Y. Bengio, "Generative Adversarial Nets," in Advances in Neural Information Processing Systems, Z. Ghahramani, M. Welling, C. Cortes, N. Lawrence, and K. Q. Weinberger, Eds., vol. 27. Curran Associates, Inc., 2014.

[9] J. Y. Zhu, T. Park, P. Isola, and A. A. Efros, "Unpaired Image-to-Image Translation Using Cycle-Consistent Adversarial Networks," Proceedings of the IEEE International Conference on Computer Vision, vol. 2017October, pp. 2242-2251, 2017.

[10] T. Karras, S. Laine, and T. Aila, "A Style-Based Generator Architecture for Generative Adversarial Networks," in 2019 IEEE/CVF Conference on Computer Vision and Pattern Recognition (CVPR), 2019, pp. 43964405.

[11] H. Zhang, T. Xu, H. Li, S. Zhang, X. Wang, X. Huang, and D. Metaxas, "StackGAN++: Realistic Image Synthesis with Stacked Generative Adversarial Networks," IEEE Transactions on Pattern Analysis and Machine Intelligence, vol. PP, 102017.

[12] H. Ye, L. Liang, G. Y. Li, and B. Juang, "Deep Learning-Based Endto-End Wireless Communication Systems With Conditional GANs as Unknown Channels," IEEE Transactions on Wireless Communications, vol. 19 , no. 5, pp. 3133-3143, 2020. 
[13] L. Sun, Y. Wang, A. L. Swindlehurst, and X. Tang, "GenerativeAdversarial-Network Enabled Signal Detection for Communication Systems With Unknown Channel Models," IEEE Journal on Selected Areas in Communications, vol. 39, no. 1, pp. 47-60, 2021.

[14] B. Hughes, S. Bothe, H. Farooq, and A. Imran, "Generative Adversarial Learning for Machine Learning empowered Self Organizing 5G Networks," in 2019 International Conference on Computing, Networking and Communications (ICNC), 2019, pp. 282-286.

[15] M. N. Fekri, A. M. Ghosh, and K. Grolinger, "Generating energy data for machine learning with recurrent generative adversarial networks," Energies, vol. 13, no. 1, pp. 1-23, 2019.

[16] S. L. Hyland, C. Esteban, and G. Rätsch, "Real-valued (medical) time series generation with recurrent conditional GANs," arXiv, 2017.

[17] O. Mogren, "C-RNN-GAN: A continuous recurrent neural network with adversarial training," in Constructive Machine Learning Workshop (CML) at NIPS 2016, 2016, p. 1.

[18] S. Takahashi, Y. Chen, and K. Tanaka-Ishii, "Modeling financial time-series with generative adversarial networks," Physica A: Statistical Mechanics and its Applications, vol. 527, p. 121261, 2019. [Online]. Available: https://www.sciencedirect.com/science/article/pii/S0378437119307277

[19] M. Wiese, R. Knobloch, R. Korn, and P. Kretschmer, "Quant GANs: deep generation of financial time series," Quantitative Finance, vol. 20, no. 9, pp. 1419-1440, 2020. [Online]. Available: https://doi.org/10.1080/14697688.2020.1730426

[20] S. Haradal, H. Hayashi, and S. Uchida, "Biosignal Data Augmentation Based on Generative Adversarial Networks," Proceedings of the Annual International Conference of the IEEE Engineering in Medicine and Biology Society, EMBS, vol. 2018-July, pp. 368-371, 2018.

[21] C. Zhang, S. R. Kuppannagari, R. Kannan, and V. K. Prasanna, "Generative Adversarial Network for Synthetic Time Series Data Generation in Smart Grids," in 2018 IEEE International Conference on Communications, Control, and Computing Technologies for Smart Grids (SmartGridComm), 2018, pp. 1-6.

[22] M. Alzantot, S. Chakraborty, and M. Srivastava, "SenseGen: A deep learning architecture for synthetic sensor data generation," in 2017 IEEE International Conference on Pervasive Computing and Communications Workshops (PerCom Workshops), 2017, pp. 188-193.

[23] C. HAN, K. MURAO, S. SATOH, and H. NAKAYAMA, "Learning More with Less: GAN-based Medical Image Augmentation," Medical Imaging Technology, vol. 37, no. 3, pp. 137-142, 2019.

[24] M. Frid-Adar, I. Diamant, E. Klang, M. Amitai, J. Goldberger, and H. Greenspan, "GAN-based synthetic medical image augmentation for increased CNN performance in liver lesion classification," Neurocomputing, vol. 321, pp. 321-331, 2018.

[25] C. Han, L. Rundo, R. Araki, Y. Furukawa, G. Mauri, H. Nakayama, and H. Hayashi, "Infinite Brain MR Images: PGGAN-Based Data Augmentation for Tumor Detection," Smart Innovation, Systems and Technologies, vol. 151, pp. 291-303, 2020.

[26] A. Shrivastava, T. Pfister, O. Tuzel, J. Susskind, W. Wang, and R. Webb, "Learning from simulated and unsupervised images through adversarial training," Proceedings - 30th IEEE Conference on Computer Vision and Pattern Recognition, CVPR 2017, vol. 2017-January, pp. 2242-2251, 2017.

[27] A. Borji, "Pros and Cons of GAN Evaluation Measures," 2018.

[28] M. Heusel, H. Ramsauer, T. Unterthiner, B. Nessler, and S. Hochreiter, "GANs Trained by a Two Time-Scale Update Rule Converge to a Local Nash Equilibrium," in Advances in Neural Information Processing Systems, I. Guyon, U. V. Luxburg, S. Bengio, H. Wallach, R. Fergus, S. Vishwanathan, and R. Garnett, Eds., vol. 30. Curran Associates, Inc., 2017.

[29] T. Salimans, I. Goodfellow, W. Zaremba, V. Cheung, A. Radford, $\mathrm{X}$. Chen, and X. Chen, "Improved Techniques for Training GANs," in Advances in Neural Information Processing Systems, D. Lee, M. Sugiyama, U. Luxburg, I. Guyon, and R. Garnett, Eds., vol. 29. Curran Associates, Inc., 2016.

[30] M. F. Naeem, S. J. Oh, Y. Uh, Y. Choi, and J. Yoo, "Reliable Fidelity and Diversity Metrics for Generative Models," in Proceedings of the 37th International Conference on Machine Learning, ser. Proceedings of Machine Learning Research, H. D. III and A. Singh, Eds., vol. 119. PMLR, 13-18 Jul 2020, pp. 7176-7185. [Online]. Available: https://proceedings.mlr.press/v119/naeem20a.html

[31] K. Shmelkov, C. Schmid, and K. Alahari, "How good is my GAN?" in Proceedings of the European Conference on Computer Vision (ECCV), September 2018.
[32] NIST/SEMATECH, "Introduction to Time Series Analysis," $e$ Handbook of Statistical Methods, 2013. [Online]. Available: https://www.itl.nist.gov/div898/handbook/pmc/section4/pmc4.htm

[33] O. Peia and K. Roszbach, "Finance and growth: Time series evidence on causality," Journal of Financial Stability, vol. 19, pp. 105-118, dec 2013.

[34] C. K. Peng, S. Havlin, H. E. Stanley, and A. L. Goldberger, "Quantification of scaling exponents and crossover phenomena in nonstationary heartbeat time series," Chaos, vol. 5, no. 1, pp. 82-87, 1995. [Online]. Available: https://pubmed.ncbi.nlm.nih.gov/11538314/

[35] A. T. Jebb, L. Tay, W. Wang, and Q. Huang, "Time series analysis for psychological research: examining and forecasting change," Frontiers in Psychology, vol. 6, p. 727, 2015. [Online]. Available: https://www.frontiersin.org/article/10.3389/fpsyg.2015.00727

[36] X. Qiu, Y. Ren, P. N. Suganthan, and G. A. Amaratunga, "Empirical Mode Decomposition based ensemble deep learning for load demand time series forecasting," Applied Soft Computing, vol. 54, pp. 246-255, 2017. [Online]. Available: https://www.sciencedirect.com/science/article/pii/S1568494617300273

[37] F. H. T. Vieira, G. R. Bianchi, and L. L. Lee, "A Network Traffic Prediction Approach Based on Multifractal Modeling," J. High Speed Netw., vol. 17, no. 2, p. 83-96, Apr. 2010.

[38] P. Cortez, M. Rio, M. Rocha, and P. Sousa, "Internet Traffic Forecasting using Neural Networks," in The 2006 IEEE International Joint Conference on Neural Network Proceedings, 2006, pp. 2635-2642.

[39] L. Kegel, M. Hahmann, and W. Lehner, "Generating WhatIf Scenarios for Time Series Data," in Proceedings of the 29th International Conference on Scientific and Statistical Database Management, ser. SSDBM '17. New York, NY, USA: Association for Computing Machinery, 2017. [Online]. Available: https://doi.org/10.1145/3085504.3085507

[40] C. Bergmeir, R. Hyndman, and J. Benítez, "Bagging exponential smoothing methods using STL decomposition and Box-Cox transformation," International Journal of Forecasting, vol. 32, no. 2, pp. 303-312, 2016. [Online]. Available: https://www.sciencedirect.com/science/article/pii/S0169207015001120

[41] A. Grover and S. Ermon, "Autoregressive models," Nov 2019. [Online]. Available: https://deepgenerativemodels.github.io/notes/autoregressive/

[42] H. Larochelle and I. Murray, "The Neural Autoregressive Distribution Estimator," in Proceedings of the Fourteenth International Conference on Artificial Intelligence and Statistics, ser. Proceedings of Machine Learning Research, G. Gordon, D. Dunson, and M. Dudík, Eds., vol. 15. Fort Lauderdale, FL, USA: PMLR, 11-13 Apr 2011, pp. 29-37. [Online]. Available: https://proceedings.mlr.press/v15/larochelle11a.html

[43] B. Uria, I. Murray, and H. Larochelle, "RNADE: The real-valued neural autoregressive density-estimator," Advances in Neural Information Processing Systems, 062013.

[44] Y. Kang, R. J. Hyndman, and F. Li, "GRATIS: GeneRAting TIme Series with diverse and controllable characteristics," Statistical Analysis and Data Mining: The ASA Data Science Journal, vol. 13, no. 4, pp. 354-376, 2020. [Online]. Available: https://onlinelibrary.wiley.com/doi/abs/10.1002/sam.11461

[45] K. Gregor, I. Danihelka, A. Mnih, C. Blundell, and D. Wierstra, "Deep AutoRegressive Networks," in Proceedings of the 31st International Conference on Machine Learning, ser. Proceedings of Machine Learning Research, E. P. Xing and T. Jebara, Eds., vol. 32, no. 2. Bejing, China: PMLR, 22-24 Jun 2014, pp. 1242-1250. [Online]. Available: https://proceedings.mlr.press/v32/gregor14.html

[46] A. van den Oord, S. Dieleman, H. Zen, K. Simonyan, O. Vinyals, A. Graves, N. Kalchbrenner, A. Senior, and K. Kavukcuoglu, "WaveNet: A Generative Model for Raw Audio," in Arxiv, 2016. [Online]. Available: https://arxiv.org/abs/1609.03499

[47] A. van den Oord, N. Kalchbrenner, O. Vinyals, L. Espeholt, A. Graves, and K. Kavukcuoglu, "Conditional Image Generation with PixelCNN Decoders," 2016.

[48] N. Patki, R. Wedge, and K. Veeramachaneni, "The Synthetic Data Vault," in 2016 IEEE International Conference on Data Science and Advanced Analytics (DSAA), 2016, pp. 399-410.

[49] Q. Wen, L. Sun, F. Yang, X. Song, J. Gao, X. Wang, and H. Xu, "Time Series Data Augmentation for Deep Learning: A Survey," 2021.

[50] J. F. Nash, "Equilibrium points in n-person games," Proceedings of the National Academy of Sciences, vol. 36, no. 1, pp. 48-49, 1950. [Online]. Available: https://www.pnas.org/content/36/1/48

[51] L. Xu, M. Skoularidou, A. Cuesta-Infante, and K. Veeramachaneni, "Modeling Tabular data using Conditional GAN," in Advances in Neural Information Processing Systems, H. Wallach, H. Larochelle, A. Beygelz- 
imer, F. d'Alché-Buc, E. Fox, and R. Garnett, Eds., vol. 32. Curran Associates, Inc., 2019.

[52] H. Chen, S. Jajodia, J. Liu, N. Park, V. Sokolov, and V. S. Subrahmanian, "FakeTables: Using GANs to Generate Functional Dependency Preserving Tables with Bounded Real Data,' in Proceedings of the Twenty-Eighth International Joint Conference on Artificial Intelligence, IJCAI-19. International Joint Conferences on Artificial Intelligence Organization, 7 2019, pp. 2074-2080. [Online]. Available: https://doi.org/10.24963/ijcai.2019/287

[53] E. Choi, S. Biswal, B. Malin, J. Duke, W. F. Stewart, and J. Sun "Generating Multi-label Discrete Patient Records using Generative Adversarial Networks," in Proceedings of the 2nd Machine Learning for Healthcare Conference, ser. Proceedings of Machine Learning Research, F. Doshi-Velez, J. Fackler, D. Kale, R. Ranganath, B. Wallace, and J. Wiens, Eds., vol. 68. PMLR, 18-19 Aug 2017, pp. 286-305. [Online]. Available: https://proceedings.mlr.press/v68/choi17a.html

[54] D. Hazra and Y.-C. Byun, "SynSigGAN: Generative Adversarial Networks for Synthetic Biomedical Signal Generation," Biology, vol. 9, no. 12, 2020. [Online]. Available: https://www.mdpi.com/2079$7737 / 9 / 12 / 441$

[55] G. M. Kimball, C. M. Pauchet, R. Ghadami, and A. F. Zaragoza, "Synthesis of multi-year PV production data using generative adversarial networks," in 2021 IEEE 48th Photovoltaic Specialists Conference (PVSC), 2021, pp. 0608-0613.

[56] J. Yoon, D. Jarrett, and M. van der Schaar, "Time-series generative adversarial networks," Advances in Neural Information Processing Systems, vol. 32, no. NeurIPS, pp. 1-11, 2019.

[57] "Using GANs for Sharing Networked Time Series Data," Proceedings of the ACM Internet Measurement Conference. [Online]. Available: http://dx.doi.org/10.1145/3419394.3423643

[58] S. Kullback and R. A. Leibler, "On Information and Sufficiency," The Annals of Mathematical Statistics, vol. 22, no. 1, pp. 79 - 86, 1951. [Online]. Available: https://doi.org/10.1214/aoms/1177729694

[59] H. Sakoe and S. Chiba, "Dynamic programming algorithm optimization for spoken word recognition," IEEE Transactions on Acoustics, Speech, and Signal Processing, vol. 26, no. 1, pp. 43-49, 1978.

[60] G. Toni, "Computing and Visualizing Dynamic Time Warping Alignments in R: The dtw Package," Journal of Statistical Software, vol. 31, 082009.

[61] H. N. Qureshi, A. Imran, and A. Abu-Dayya, "Enhanced MDT-Based Performance Estimation for AI Driven Optimization in Future Cellular Networks," IEEE Access, vol. 8, pp. 161 406-161 426, 2020.

[62] F. Pedregosa, G. Varoquaux, A. Gramfort, V. Michel, B. Thirion, O. Grisel, M. Blondel, P. Prettenhofer, R. Weiss, V. Dubourg, J. Vanderplas, A. Passos, D. Cournapeau, M. Brucher, M. Perrot, and E. Duchesnay, "Scikit-learn: Machine Learning in Python," Journal of Machine Learning Research, vol. 12, pp. 2825-2830, 2011.

[63] Y. Luo, X. Cai, Y. ZHANG, J. Xu, and Y. xiaojie, "Multivariate Time Series Imputation with Generative Adversarial Networks," in Advances in Neural Information Processing Systems, S. Bengio, H. Wallach, H. Larochelle, K. Grauman, N. Cesa-Bianchi, and R. Garnett, Eds., vol. 31. Curran Associates, Inc., 2018.

[64] J. Yoon, J. Jordon, and M. van der Schaar, "GAIN: Missing Data Imputation using Generative Adversarial Nets," in Proceedings of the 35th International Conference on Machine Learning, ser Proceedings of Machine Learning Research, J. Dy and A. Krause, Eds., vol. 80. PMLR, 10-15 Jul 2018, pp. 5689-5698. [Online]. Available: https://proceedings.mlr.press/v80/yoon18a.html 